\title{
Espalhamento inelástico da luz por portadores no plasma em semicondutores. II Cálculo do Espectro Raman
}

\author{
Light Scattering by Carriers Plasmas in Semiconductors. II Raman Scattering Analysis
}

\author{
A.V. Andrade-Neto* \\ Departamento de Física - UEFS \\ Campus Universitário $\mathrm{Km} 03 \mathrm{BR} 116$ \\ Feira de Santana - BA - 44031-460
}

\begin{abstract}
Neste trabalho é discutido o espalhamento inelástico da luz por portadores no plasma simples em semicondutores. Calculamos a seção de choque do espalhamento Raman, a qual está diretamente relacionada, via teorema de flutuação-dissipação, com a função dielétrica do sistema. Nossos cálculos são comparados com resultados experimentais do GaAs nos quais são evidenciados o amortecimento de Landau.
\end{abstract}

Palavras-chaves: Espalhamento Raman, Teorema de flutuação-dissipação, Amortecimento de Landau.

In this work we obtain, using fluctuation-dissipation theorem, the Raman scattering for singlecomponent plasma.We find that the dynamical structure factor, hence the light scattering spectra, are directly related to imaginary part of the inverse of the dielectric function. We compare our results with measured of n-type GaAs where the modes in the electron gas are single-particle excitations as the plasmon is Landau damped.

Key-words: Raman Scattering, Fluctuation-Dissipation theorem, Landau Dampimg.

\section{INTRODUÇÃO}

O espalhamento inelástico da luz por vibrações cristalinas ou moleculares é denominado Espalhamento Raman e tem como causa a modulação da polarizabilidade do meio pelas vibrações [1]. O espalhamento Raman em sólidos causado por outras excitações elementares como, por exemplo, o plasmon ocorre pelo mesmo mecanismo. Este efeito foi previsto teoricamente por A. Smekal em 1923 e verificado experimentalmente alguns anos depois (1928) por C. V. Raman, o qual foi agraciado com o prêmio Nobel em 1930 por essa descoberta. Entretanto, a falta de uma fonte de luz coerente dificultava esse estudo, o que mudou apenas com a invenção do laser na década de 1960, sendo o físico Brasileiro Sérgio P. S. Porto um pioneiro no uso da radiação de lasers na espectroscopia Raman. Essa área experimental engloba experimentos integrados no tempo ou experimentos com resolução temporal ultra-rápida. Aqui estamos interessados nos primeiros.

Em particular, as propriedades ópticas de materiais semicondutores têm sido extensivamente estudadas, tanto do ponto de vista teórico quanto experimental. Esse grande interesse se justifica, por um lado, na busca por maior compreensão de suas propriedades microscópicas - já que há disponível um grande número de dados experimentais de ótima qualidade - e, por outro, por suas enormes aplicações tecnológicas, uma vez que o modo de vida contemporâneo é indissociável da revolução microeletrônica, cuja base são os materiais semicondutores.

Uma quantidade física fundamental a ser determinada no estudo das propriedades ópticas de sólidos é a função dielétrica, a qual descreve a resposta do sistema a uma per-

*Endereço Eletrônico: aneto@uefs.br turbação elétrica externa. O seu conhecimento é de fundamental importância porque, como vimos no Artigo I, quantidades físicas determinadas experimentalmente, tais como coeficiente de absorção, a refletividade, o espectro Raman, etc, podem ser calculadas a partir da função dielétrica, bem como fornece informações a respeito das excitações elementares do sistema (de quasi-partículas individuais e oscilações coletivas). Neste artigo, em particular, calcularemos o espectro Raman.

Nos experimentos de espalhamento utilizados para estudar plasma em estado sólido, o feixe incidente pode consistir de elétrons rápidos ou radiação eletromagnética de várias freqüências (infravermelho, visível, raios X). Contudo, para semicondutores, a oscilação de plasma, $\left[\omega_{p}=\right.$ $\left.\sqrt{4 \pi e^{2} n /\left(\epsilon_{\infty} m^{*}\right)}\right]$, corresponde a energias demasiadas pequenas para serem convenientemente detectadas em um experimento de espalhamento de elétrons [2]. Por esse motivo, espalhamento inelástico da luz é muito utilizado para estudar essas excitações, particularmente após a invenção do laser.

Podemos subdividir as freqüências espalhadas em duas categorias: a banda de freqüência menor que a incidente, conhecida como componente Stokes; e a banda de freqüência maior, que forma a componente anti-Stokes. Em ambas as situações o espalhamento é causado por flutuações de densidade no sistema de elétrons. Essas flutuações podem estar associadas ao comportamento de partículas individuais ou a modos coletivos do mesmo. Qualquer que seja o caso, existe um espectro de freqüências nos fótons espalhados que fornece informações importantes acerca da estrutura do sistema de muitos corpos.

As primeiras observações de espalhamento inelástico da luz por plasma em estado sólido foram realizadas na década de 1960 [3], e desde então tem sido objeto de interese de diversos pesquisadores e tem merecido especial atenção do grupo de Mecânica Estatística de Sistemas Dissipativos do Instituto de Física da UNICAMP [4]. Como já foi dito, o es- 
pectro Raman fornece informações fundamentais a respeito das excitações elementares do sistema em estudo, tanto de partículas individuais quanto de oscilações coletivas.

\section{ACOPLAMENTO ELÉTRON-FÓTON}

Consideremos um sistema de portadores individuais de carga $-e$ e massa efetiva $m^{*}$, movimentando-se num fundo de cargas positivas (os íons) de maneira que o sistema seja globalmente neutro. Este sistema é submetido a um campo eletromagnético caracterizado pelo potencial vetor $\vec{A}$, que em mecânica quântica representa o campo de radiação quantizado.

O hamiltoniano que descreve a interação da radiação incidente e espalhada com os elétrons pode ser escrito como

$$
\hat{\mathcal{H}}=\sum_{j} \frac{1}{2 m^{*}}\left(\hat{p}_{j}-\frac{e}{c} \hat{A}\left(\vec{x}_{j}\right)\right)^{2}+\hat{H}^{\prime}
$$

onde $c$ é a velocidade da luz, $\hat{p}_{j}$ é o operador momentum do j-ésimo portador na posição $\vec{x}_{j}, \hat{A}\left(\vec{x}_{j}\right)$ representa a soma do potencial vetor do campo incidente e espalhado com freqüências $\omega_{0}$ e $\omega_{1}$, respectivamente e $\hat{H}^{\prime}$ descreve a interação entre os elétrons e entre estes e a rede.

Podemos reescrever o hamiltoniano (1) na forma

$$
\begin{aligned}
\hat{\mathcal{H}}= & \sum_{j} \frac{\hat{p}_{j}^{2}}{2 m^{*}}+\hat{H}^{\prime}+\frac{e^{2}}{2 m^{*} c^{2}} \sum_{j} \hat{A}^{2}\left(\vec{x}_{j}\right) \\
& -\frac{e}{2 m^{*} c} \sum_{j}\left(\hat{p}_{j} \hat{A}\left(\vec{x}_{j}\right)+\hat{A}\left(\vec{x}_{j}\right) \hat{p}_{j}\right)
\end{aligned}
$$

mas, sabemos que

$$
\sum_{i=1}^{3}\left[p_{i}, A_{i}\right]_{-}=-\imath \hbar \sum_{i} \frac{\partial A_{i}}{\partial x_{i}}=-\imath \hbar \operatorname{div} \vec{A}
$$

onde $[,]_{-}$indica comutador, então

$$
\hat{p} \cdot \hat{A}=[\hat{p}, \hat{A}]_{-}+\hat{A} \cdot \hat{p}=-\imath \hbar \operatorname{div} \vec{A}+\hat{A} \cdot \hat{p}
$$

Utilizando (4) em (2) e utilizando o chamado gauge de Coulomb (i.e., $\operatorname{div} \vec{A}=0$ ), podemos escrever o hamiltoniano do sistema na seguinte forma

$$
\hat{\mathcal{H}}=\hat{H}+\hat{H}_{1}+\hat{H}_{2}
$$

onde

$$
\hat{H}=\sum_{j} \frac{\hat{p}_{j}^{2}}{2 m^{*}}+\hat{H}^{\prime}
$$

é o hamiltoniano não perturbado,

$$
\hat{H}_{1}=-\frac{e}{m^{*} c} \sum_{j} \hat{A}\left(\vec{x}_{j}\right) \cdot \hat{p}_{j}
$$

é o acoplamento linear portador-radiação e

$$
\hat{H}_{2}=\frac{e^{2}}{2 m^{*} c^{2}} \sum_{j} \hat{A}^{2}\left(\vec{x}_{j}\right)
$$

é o termo quadrático do acoplamento elétron-fóton.
Vemos, assim, que temos dois tipos de acoplamento portador-radiação: um termo linear $\hat{A} \cdot \hat{p}$ e um termo quadrático $\hat{A}^{2}$. De modo geral, a interação elétronfóton provoca transições interbandas ou intrabanda. A transição interbanda, da banda de valência para a banda de condução, ocorre quando a energia da radiação está próxima da transição eletrônica permitida. Por sua vez, transições eletrônicas intrabanda ocorrem quando a energia dos fótons incidentes $\left(\hbar \omega_{0}\right)$ é menor e não próximo do intervalo da banda proibida $\left(E_{G}\right)$, ou seja, em condições afastadas do caso de ressonância. Este último caso ocorre, por exemplo, em semicondutores dopados que é o sistema que estamos interessados neste artigo.

Para a situação onde as transições eletrônicas são intrabanda, pode-se mostrar [2, 5] que o termo linear - (que produz espalhamento em segunda ordem), comparado com o quadrático - tem uma contribuição muito pequena para a seção de choque do espalhamento da luz (da ordem de $(v / c)^{2}$ onde $v$ é a velocidade média dos portadores, velocidade de Fermi a baixas temperaturas ou velocidade térmica a altas temperaturas). Então, a quase totalidade do espalhamento vem do termo quadrático $A_{j}^{2}$. A expressão para a seção de espalhamento é dada a seguir.

\section{SEÇÃO DE ESPALHAMENTO}

A seção de choque diferencial para espalhamento inelástico da luz, por intervalo de freqüência $d \omega$ e intervalo de ângulo sólido $d \Omega$ é dada por [2]

$$
\frac{d^{2} \sigma}{d \omega d \Omega}=r_{o}^{2}\left(\frac{\omega_{1}}{\omega_{0}}\right)\left(\hat{e}_{0} \cdot \hat{e}_{1}\right)^{2} S(\vec{Q}, \omega)
$$

onde

$$
\omega=\omega_{0}-\omega_{1}
$$

é a freqüência de espalhamento ${ }^{1} \mathrm{e}$

$$
Q=k_{0}-k_{1}
$$

representa, em unidades de $\hbar$ o momento transferido no processo e

$$
r_{o}=\frac{e^{2}}{m^{*} c^{2}} \sim,
$$

é o raio clássico do elétron.

Finalmente $S(\vec{Q}, \omega)$ é o chamado fator de estrutura dinâmico definido, em termos da função correlação densidade-densidade, como

$$
S(\vec{Q}, \omega)=\frac{1}{2 \pi} \int_{-\infty}^{\infty} d t \exp (\imath \omega t)<\hat{n}_{\vec{Q}}(t)\left(\hat{n}_{\vec{Q}}\right)^{\dagger}(0)>\sim
$$

onde $\langle\ldots\rangle$ representa a média sobre o ensemble apropriado à descrição do estado macroscópico do sistema no momento do experimento e $\hat{n}_{\vec{Q}}(t)$ é a transformada de Fourier do operador densidade de partícula, $\hat{n}(\vec{x})$, na representação de Heisenberg, i.e.,

$$
\hat{n}_{\vec{Q}}(t)=\exp \left(\frac{\imath \hat{H}}{\hbar} t\right) \hat{n}_{\vec{Q}} \exp \left(-\frac{\imath \hat{H}}{\hbar} t\right)
$$

onde $\hat{H}$ é o hamiltoniano do sistema de muitos corpos.

\footnotetext{
1 Em unidades de $\hbar$ é a energia transferida no processo.
} 


\section{TEOREMA DE FLUTUAÇÃO-DISSIPAÇÃO}

Como vimos Eq.(9), a quantidade física fundamental que determina a seção de choque de espalhamento é o fator de estrutura dinâmico Eq.(13). Esta formulação é bastante conveniente porque, como mostraremos a seguir, o fator de estrutura dinâmico $S(\vec{Q}, \omega)$ está diretamente relacionado com a função dielétrica do sistema. Esta relação se obtém via teorema de flutuação-dissipação que é um resultado muito comum em Física Estatística.

A função dielétrica do sistema é dada pela seguinte expressão geral [6]

$$
\epsilon^{-1}(\vec{Q}, \omega)=1+2 \pi V(Q) \ll \hat{n}_{\vec{Q}} \mid \hat{n}_{-\vec{Q}} ; \tilde{\omega} \gg
$$

onde $V(Q)=4 \pi e^{2} /\left(\mathcal{V} Q^{2}\right)$ é a transformada de Fourier do potencial coulombiano e

$$
\begin{aligned}
\ll \hat{n}_{\vec{Q}} \mid \hat{n}_{-\vec{Q}} ; \omega \gg= & \frac{1}{2 \pi} \int_{-\infty}^{\infty} d t e^{\imath(\omega+\imath s) t} \frac{\theta(t)}{\imath \hbar} \times \\
& \times\left\langle\left[\hat{n}_{\vec{Q}}(t), \hat{n}_{-\vec{Q}}(0)\right]_{-}\right\rangle
\end{aligned}
$$

é a transformada de Fourier da função de Green de tempo duplo [7] da função resposta densidade-densidade onde $s$ é uma constante infinitesimal positiva, $\theta(t)$ é a função degrau de Heaviside e $\hat{n}_{\vec{Q}}$ é dado por (14).

Das equações (13) e (15) vemos que existe uma estreita relação entre $S(\vec{Q}, \omega)$ e a função de Green da correlação densidade-densidade.

Para determinar como essas duas quantidades estão relacionadas, vamos supor que conhecemos um conjunto completo de estados do sistema interagente.

Assumiremos que o sistema obedece a uma distribuição canônica, i.e.

$$
<\ldots>=\frac{1}{Z} \operatorname{Tr}\left(e^{-\beta \hat{H}} \ldots\right)=\frac{1}{Z} \sum_{m} e^{-\beta E_{m}}<m|\ldots| m>
$$

com $\beta=1 /\left(k_{B} T\right)$ onde $k_{B}$ é a constante de Boltzmann e $T$ é a temperatura do sistema, $Z$ é a função partição do sistema e $\mid m>$ é um conjunto completo de funções ortonormais.

Utilizando a média acima em (13) temos que

$$
\begin{aligned}
S(\vec{Q}, \omega)= & \frac{1}{2 \pi Z} \sum_{m} e^{-\beta E_{m}} \int_{-\infty}^{\infty} d t e^{\imath \omega t}<m\left|\hat{n}_{\vec{Q}}(t) \hat{n}_{-\vec{Q}}(0)\right| m> \\
& =\frac{1}{2 \pi Z} \sum_{m, m^{\prime}} e^{-\beta E_{m}} \int_{-\infty}^{\infty} d t e^{\imath \omega t}<m\left|\hat{n}_{\vec{Q}^{(}}(t)\right| m^{\prime}><m^{\prime}\left|\hat{n}_{-\vec{Q}}(0)\right| m> \\
& =\frac{1}{2 \pi Z} \sum_{m, m^{\prime}} e^{-\beta E_{m}} \int_{-\infty}^{\infty} d t e^{\imath \omega t}\left(<m\left|e^{\imath \hat{H} t / \hbar} \hat{n}_{\vec{Q}} e^{-\imath \hat{H} t / \hbar}\right| m^{\prime}>\right) \\
& \times\left(<m^{\prime} \mid \hat{n}_{\left.\left.-\vec{Q}^{(}\right) \mid m>\right) .}(0)\right.
\end{aligned}
$$

Utilizando que

$$
e^{\imath \hat{H} t / \hbar}\left|m>=e^{\imath E_{m} t / \hbar}\right| m>
$$

e depois de algumas simplificações obtemos

$$
\begin{aligned}
S(\vec{Q}, \omega)= & \frac{1}{Z} \sum_{m, m^{\prime}} e^{-\beta E_{m}}|<m| \hat{n}_{\vec{Q}}\left|m^{\prime}>\right|^{2} \times \\
& \times \delta\left(\omega+\frac{E_{m}}{\hbar}-\frac{E_{m^{\prime}}}{\hbar}\right)
\end{aligned}
$$

onde

$$
\begin{aligned}
& \delta\left(\omega+\frac{E_{m}}{\hbar}-\frac{E_{m^{\prime}}}{\hbar}\right) \\
& =\frac{1}{2 \pi} \int_{-\infty}^{\infty} d t \exp \left[\imath\left(\omega+\frac{E_{m}}{\hbar}-\frac{E_{m^{\prime}}}{\hbar}\right) t\right] .
\end{aligned}
$$

De modo similar podemos mostrar que

$$
\begin{aligned}
T(\vec{Q}, \omega)= & \frac{1}{2 \pi} \int_{-\infty}^{\infty} d t e^{\imath \omega t}<\hat{n}_{-\vec{Q}}(0) \hat{n}_{\vec{Q}}(t)> \\
& =\frac{1}{Z} \sum_{m, m^{\prime}} e^{-\beta E_{m}}|<m| \hat{n}_{-\vec{Q}}\left|m^{\prime}>\right|^{2} \times \\
& \times \delta\left(\omega+\frac{E_{m^{\prime}}}{\hbar}-\frac{E_{m}}{\hbar}\right)
\end{aligned}
$$

como $m$ e $m^{\prime}$ são índices mudos podemos invertê-los em (22) e obtemos depois de alguns cálculos

$$
T(\vec{Q}, \omega)=e^{-\beta \hbar \omega} S(\vec{Q}, \omega) .
$$

Segue da Eq.(13) que

$$
<\hat{n}_{\vec{Q}}(t) \hat{n}_{-\vec{Q}}(0)>=\int_{-\infty}^{\infty} d \omega e^{-\imath \omega t} S(\vec{Q}, \omega)
$$

$$
<\hat{n}_{-\vec{Q}}(0) \hat{n}_{\vec{Q}}(t)>=\int_{-\infty}^{\infty} d \omega e^{-\imath \omega t} T(\vec{Q}, \omega) .
$$

Utilizando (24) e (25) em (16) obtemos

$$
\begin{aligned}
\ll \hat{n}_{\vec{Q}} \mid \hat{n}_{-\vec{Q}} ; \omega \gg & =\frac{1}{2 \pi \imath \hbar} \int_{-\infty}^{\infty} d \omega^{\prime}\left[S\left(\vec{Q}, \omega^{\prime}\right)-T\left(\vec{Q}, \omega^{\prime}\right)\right] \times \\
& \times \int_{0}^{\infty} d t e^{\imath\left(\omega-\omega^{\prime}+\imath s\right) t},
\end{aligned}
$$

mas

$$
\int_{0}^{\infty} d t e^{\imath\left(\omega-\omega^{\prime}+\imath s\right) t}=\frac{-1}{\imath\left(\omega-\omega^{\prime}+\imath s\right)}
$$


então

$$
\begin{aligned}
\ll \hat{n}_{\vec{Q}} \mid \hat{n}_{-\vec{Q}} ; \tilde{\omega} \gg & =\frac{1}{2 \pi \hbar} \int_{-\infty}^{\infty} d \omega^{\prime} \frac{\left[S\left(\vec{Q}, \omega^{\prime}\right)-T\left(\vec{Q}, \omega^{\prime}\right)\right]}{\omega-\omega^{\prime}+\imath s} \\
& =\frac{1}{2 \pi \hbar} \int_{-\infty}^{\infty} d \omega^{\prime} \frac{\left[\left(1-e^{-\beta \hbar \omega^{\prime}}\right) S\left(\vec{Q}, \omega^{\prime}\right)\right]}{\omega-\omega^{\prime}+\imath s},
\end{aligned}
$$

onde usamos (23).

Usando a identidade

$$
\lim _{s \rightarrow 0} \frac{1}{X \pm i s}=v \cdot p \cdot \frac{1}{X} \mp i \pi \delta(X)
$$

onde v.p. indica o valor principal de uma integral na qual esta relação é usada e $\delta(X)$ é a função delta de Dirac, obtemos

$$
\begin{aligned}
& \ll \hat{n}_{\vec{Q}} \mid \hat{n}_{-\vec{Q}} ; \tilde{\omega} \gg \\
= & \frac{1}{2 \pi \hbar} v \cdot p \cdot \int_{-\infty}^{\infty} d \omega^{\prime} \frac{1-e^{-\beta \hbar \omega^{\prime}}}{\omega-\omega^{\prime}} S(\vec{Q}, \omega) \\
& -\frac{\imath}{2 \hbar} \int_{-\infty}^{\infty} d \omega^{\prime}\left(1-e^{-\beta \hbar \omega^{\prime}}\right) S\left(\vec{Q}, \omega^{\prime}\right) \delta\left(\omega-\omega^{\prime}\right) .
\end{aligned}
$$

Logo

$$
\operatorname{Im}\left[\ll \hat{n}_{\vec{Q}} \mid \hat{n}_{-\vec{Q}} ; \tilde{\omega} \gg\right]=\frac{1}{2 \hbar}\left(e^{-\beta \hbar \omega}-1\right) S(\vec{Q}, \omega)
$$

onde $I m$ representa parte imaginária. Mas, de (15) temos que

$$
\operatorname{Im}\left[\ll \hat{n}_{\vec{Q}} \mid \hat{n}_{-\vec{Q}} ; \tilde{\omega} \gg\right]=\frac{1}{2 \pi V(Q)} \operatorname{Im}\left[\epsilon^{-1}(\vec{Q}, \omega)\right] .
$$

De (31) e (32) resulta que

$$
S(\vec{Q}, \omega)=\hbar[1-\exp (-\beta \hbar \omega)]^{-1} \frac{1}{\pi V(Q)} \operatorname{Im}\left[\frac{-1}{\epsilon(\vec{Q}, \omega)}\right] .
$$

A (33) relaciona $S(\vec{Q}, \omega)$, que governa a flutuação na densidade dos portadores, com a parte imaginária de $\epsilon(\vec{Q}, \omega)$, que governa a dissipação no sistema; sendo, portanto, uma das formas do teorema de flutuação-dissipação.

Utilizando (33) em (9) obtemos para a seção de espalhamento

$$
\frac{d^{2} \sigma}{d \omega d \Omega} \sim[1-\exp (-\beta \hbar \omega)]^{-1} \operatorname{Im}\left[\frac{-1}{\epsilon(\vec{Q}, \omega)}\right]
$$

que é a relação a ser utilizada.

\section{COMPARAÇÃO COM RESULTADOS EXPERIMENTAIS}

Apresentaremos agora alguns resultados experimentais disponíveis para o GaAs.

Na Figura 1 (retirada da referência [3]) mostra-se o espectro Raman do GaAs para uma concentração $n=10^{16} \mathrm{~cm}^{-3}$ para várias temperaturas. Podemos identificar claramente duas regiões distintas: uma região no intervalo $\pm 100 \mathrm{~cm}^{-1}$ causado por elétrons e outra região entre 250 e $300 \mathrm{~cm}^{-1}$ devido aos fônons TO e LO. Neste trabalho estamos interessados na parte do espectro Raman devido aos elétrons, i.e., no intervalo $\pm 100 \mathrm{~cm}^{-1}$.

É dito na referência [3]) que o valor do número de onda transferido é igual ao inverso do comprimento de blindagem à temperatura ambiente, então usaremos que $Q=k_{D H}=2,3 \times 10^{5} \mathrm{~cm}^{-1}$ [conforme Eq.(32) do Artigo I]. Para a concentração utilizada a temperatura de Fermi é $T_{F}=29,3 K$, então, com exceção do caso $T=5 K$, podemos usar os resultados obtidos no Artigo I para a função dielétrica e esta na Eq.(34) para encontrarmos a seção de espalhamento. As Figuras 2, 3 e 4 mostram os nossos resultados para, respectivamente, $T=300,150$ e $50 K$. Dos resultados experimentais e dos nossos cáculos vemos nitidamente que, com o aumento da temperatura, a banda associada ao modo de plasma vai ficando menos definida, i.e., tende a ficar submergida na banda de partículas individuais o que evidencia o amortecimento de Landau (Landau Damping). Vemos também que a altura do pico da banda associada ao modo de plasma na componente Stokes é maior do que na componente anti-Stokes, e que esta diferença também se intensifica com a diminuição da temperatura. Esta assimetria se deve ao fato de que é muito mais provável os fótons cederem energia aos portadores do que estes cederem energia aos fótons; e mais, a probabilidade de ocorrer a primeira situação (os fótons cederem energia) aumenta com a diminuição da temperatura já que, quanto menor a temperatura menor será o número de elétrons em estados excitados, o que explica o comportamento do espectro Raman com a temperatura.

Outro efeito visível nas figuras é que com a diminuição da temperatura as linhas de plasma (Stokes e anti-Stokes) tornam-se cada vez mais nítidas, com o consequente predomínio dessa linha no espalhamento da luz. Isso ocorre porque com a diminuição da temperatura, mantendo a concentração constante, o vetor de onda de Debye-Hückel $\left(k_{D H}\right)$ aumenta; então o momemtum transferido $(\hbar Q)$ é cada vez menor em comparação com $\hbar k_{D H}$ e nesta situação a banda correspondente ao plasmon é muito intensa.

\section{COMENTÁRIOS FINAIS}

Discutimos neste trabalho o espalhamento inelástico da luz por portadores no plasma em semicondutores. Inicialmente (no Artigo I) calculamos a função dielétrica do sistema no limite de altas temperaturas $T \gg T_{F}$. No Artigo II calculamos o espectro Raman para o GaAs e comparamos nossos resultados com resultados experimentais disponíveis para este material. Os nossos cálculos reproduzem o comportamento do subsistema eletrônico de forma muito boa. Em particular vemos que, mantendo a concentração fixa, com o aumento da temperatura diminue o predomínio da banda associada ao modo de plasma em comparação com a banda de partículas individuais, o que ilustra de forma muito clara o amortecimento de Landau. 


$$
\text { GaAs, } n=1 \times 10^{16} \mathrm{~cm}^{-3}
$$

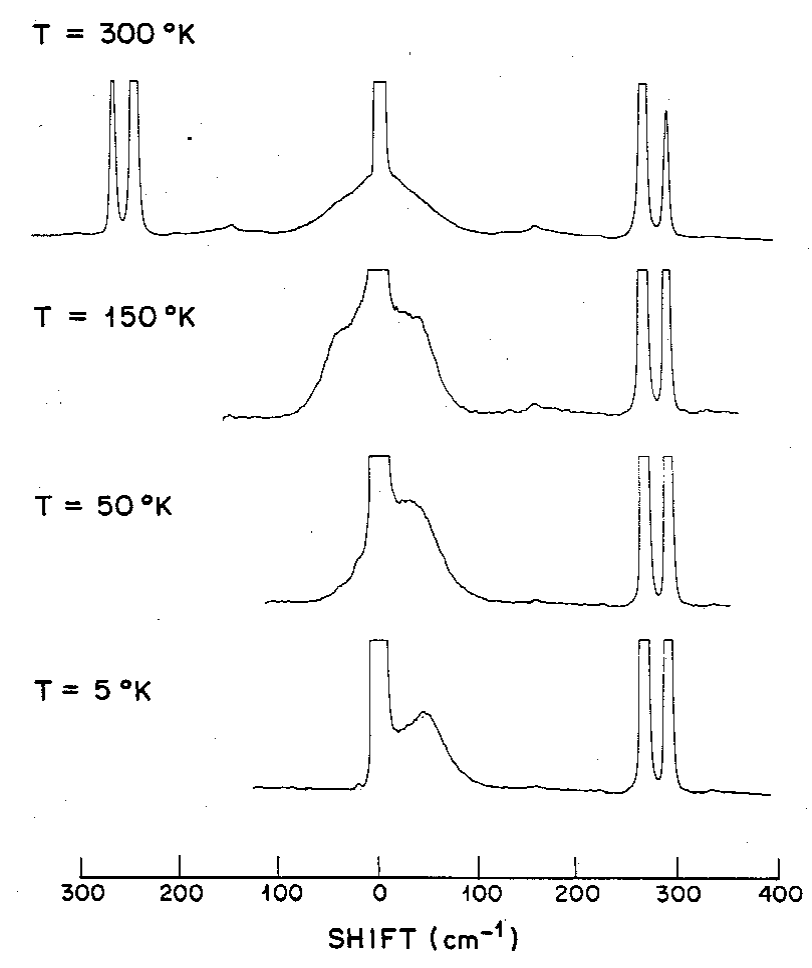

Fig. 1 - Espectro Raman do GaAs para $n=10^{16} \mathrm{~cm}^{-3}$.

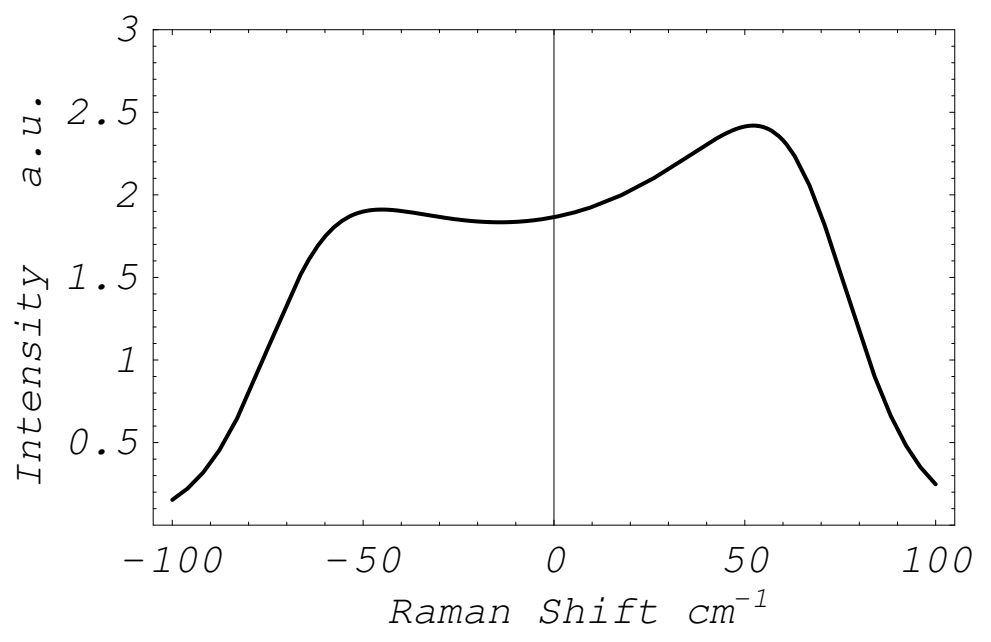

Fig. 2 - Espectro Raman do GaAs para $n=10^{16} \mathrm{~cm}^{-3}$ e $T=300 \mathrm{~K}\left(k_{D H}=2.5 \times 10^{5} \mathrm{~cm}^{-1}\right)$.

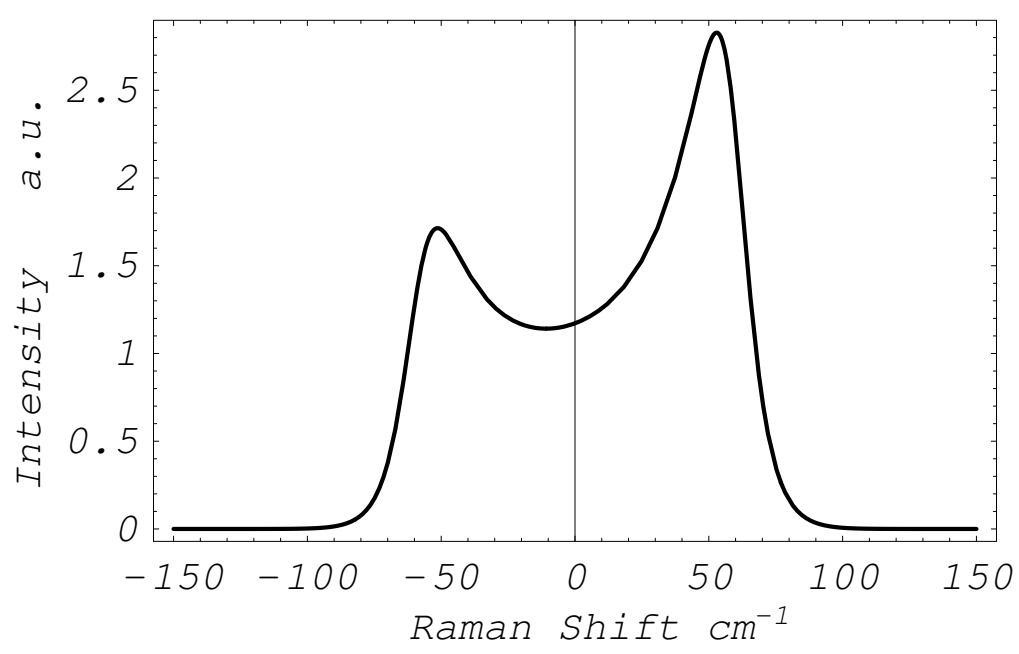


Fig. 3 - Espectro Raman do GaAs para $n=10^{16} \mathrm{~cm}^{-3}$ e $T=150 \mathrm{~K}\left(k_{D H}=3.6 \times 10^{5} \mathrm{~cm}^{-1}\right)$.

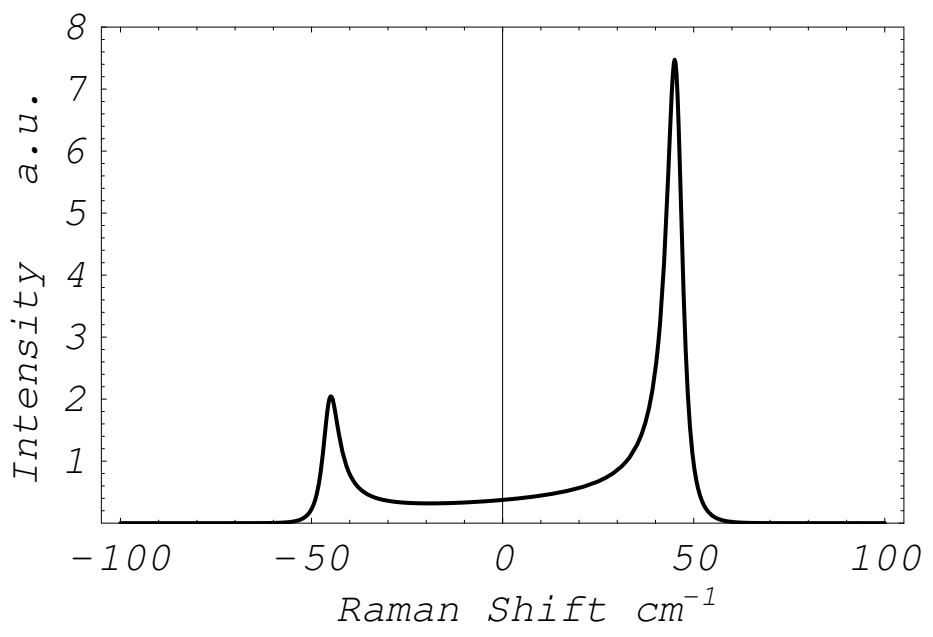

Fig. 4 - Espectro Raman do GaAs para $n=10^{16} \mathrm{~cm}^{-3}$ e $T=50 \mathrm{~K}\left(k_{D H}=6.2 \times 10^{5} \mathrm{~cm}^{-1}\right)$.

[1] W. Hayes and R. Loudon, Scattering of Light by Cristals, John Wiley, New York (1978).

[2] P. M. Platzman and P. A. Wolff, Waves and Interactions in Solid State Plasmas In F. Seitz, D. Turnbull and H. Ehrenrich editors, Solid State Phys., Suppl. 13, Academic Press, New York (1973).

[3] A. Mooradian and A. L. MacWhorter. Light Scattering from plasmons and phonons in GaAs. In G. B. Wright, editor Light Scattering Spectra of Solids, Springer, New York (1969).

[4] R. Luzzi, A. R. Vasconcellos and J. G. P. Ramos, Predictive Statistical Mechanics: A Nonequilibrium Ensemble Formal- ism, Kluwer Academic Dordrecht (2002)

[5] G. Abstreiter, M. Cardona and A. Pinczuk. Light Scattering in Solids IV, Editors: M. Cardona and G. Güntherodt 54, 1 (1984).

[6] Antônio Vieira de Ancrade Neto, Estudo dos Efeitos Polares e de Muitos Corpos nas Propriedades Óticas de IIINitretos. Tese de Doutorado - Unicamp - (2005).

[7] D. N. Zubarev, Nonequilibrium Statistical Thermodinamics, Consultants Bureau, New York (1974).

[8] S. Nakajimal et all, The Physics of Elementary Excitations, Springer-Verlag, Berlin (1980). 